\title{
Design Sensitive Model Reference Controller with Application to Medical Technology
}

\author{
${ }^{1}$ Rouhollah Bahrami, ${ }^{1}$ Samira Soltani, ${ }^{1}$ Hossein Rashidi Bod, ${ }^{1}$ Somayeh Jowkar, \\ ${ }^{1}$ Amirzubir Sahamijoo, ${ }^{1}$ Ali Taghizadegan ${ }^{1,2}$ and Nasri. B Sulaiman \\ ${ }^{1}$ Intelligent System and Robotic Lab, Iranian Institute of Advanced Science and \\ Technology (IRAN SSP), Shiraz/Iran \\ ${ }^{2}$ Department of Electrical Engineering, Faculty of Engineering, University Putra \\ Malaysia, Malaysia \\ info@iranssp.org,www.iranssp.org/english
}

\begin{abstract}
The past two decades have seen the incorporation of robotics into medical applications. From a manufacturing perspective, robots have been used in pharmaceuticals, preparing medications. But on more novel levels, robots have been used in service roles, surgery, and prosthetics. The capability of high-precision operation in manufacturing settings gave the medical industry high hopes that robots could be used to assist in surgery. Not only are robots capable of much higher precision than a human, they are not susceptible to human factors, such as trembling and sneezing, that are undesirable in the surgery room. Another advantage to robots in medicine is the ability to perform surgery with very small incisions, which results in minimal scar tissue, and dramatically reduced recovery times. The popularity of these minimally invasive surgical (MIS) procedures has enabled the incorporation of robots in surgeries.

In this paper, model reference computed torque controller is recommended for four degrees of freedom serial links robot manipulator, which modeled in SIMSCAPE. To design stable controller conventional computed torque controller is recommended. It is a nonlinear, stable, and reliable controller. The proposed approach effectively combines of design methods from nonlinear controller, and linear Proportional-Derivative (PD) control to improve the performance and stability. This paper has two important objectives: a) study on modeling of 4 degrees of freedom (DOF) based on Simscape software and b) design PD model reference computed torque controller to improve the sensitivity of surgical robot manipulator.
\end{abstract}

Keywords: surgical robots, four degrees of freedom, model reference computed torque controller

\section{Introduction}

Before one can consider the usage of robots in medical applications, it is important to understand that medical applications have unique requirements, different from general, or "traditional," robot design.

Some of the design issues and associated advantages are described below.

High precision: Modern robots are demonstrated to be highly precise. The precision range depends on the robot and the application, of course, but it is generally accepted that for a given application, a robot can be designed to meet or exceed the precision requirements of the application. A typical industrial robot has repeatability specifications measured in tenths of a millimeter. A representative ratio of motion in robotic assisted surgery is that a $1 \mathrm{~cm}$ movement of a doctor's hand translates to a $0.1 \mathrm{~cm}$ movement of the robotic tool. 
Heavy payloads: Modern robots can carry heavy payloads over large workspaces, at high speeds, with high precision. Industrial robots are available with payload capacity of a few ounces to over $1000 \mathrm{lb}$.

Workspace: Medical robot workspace requirements tend to be significantly larger than industrial needs because of patient related factors, such as uncertainty in patient location during the procedure and safety requirements. There is an obvious overriding need to avoid any hazard to the patient, physician, and other medical personnel; this drives an exclusionary zone around the patient, doctor, and other equipment that may be attached to the patient. Thanks to the advances driven by industrial applications, the workspace of most available robots is significant and can be utilized for medical applications.

High Speed: Most new robots have been designed and optimized for industrial automation, enabling them to move at high speeds with high precision. The majority of medical applications do not require robots to move at high speeds as these robots are working on a patient. Reassurance, comfort, and safety dictate the robot's speed in medical applications.

Reliability: Industrial robots are designed to work round the clock without stopping; their medical counter parts work only a few hours a day. The nature of medical applications is that most of the time

is taken up by other parts of the surgery, such as operating room preparation, patient preparation, and postoperative procedures. The robots actually perform surgeries for only a limited time, around $10 \%$ of surgery time. The resulting reliability numbers for medical work are excellent, leading to very limited downtime.

Tedium: Most of the medical applications where robots are sought involve repetitive tasks over a very long period of time. Some surgeries last for many hours, during which the operators are required to repeat tasks hundreds or thousands of times. Obviously, robots do not have any problems with tedium.

High Quality: Robotic assisted surgery can help a wide variety of doctors perform complex surgeries with the same high quality previously achieved only by some accomplished surgeons. Additionally, most medical procedures cannot tolerate any degradation in quality due to trembling or unsteadiness of hands. Robotic systems in the operating room can compensate for imperfections in the user due to age, fatigue, or other factors, without degrading the quality of care administered to the patient.

Computer control: Robotic surgery is able to capitalize on available diagnostic data to calculate an optimized approach to treatment. Most modern systems use fusion of multiple imaging modalities such as CT, PET, and MRI.

Remote operation: Finally, because robots are typically controlled by computers and/or remote electrical signals, the option exists to remotely operate the units over large distances through direct data links, or even over the internet (telerobotics).

Robot-assisted surgery has become a burgeoning field in recent years. An interdisciplinary subject involves both robot technologies and medical intervention. Because of its potentials to improve precision, enhance dexterity, eliminate tremor, reduce complication rates, and enable novel procedures not previously achievable, robot-assisted surgery has drawn broad attentions from the robotics research community and the medical world over the past decade. One successful implementation of robot-assisted surgery is the DAVINCI surgical system of Intuitive Surgical. It utilizes a tele-operation control mode with a master controlled by the surgeon and a slave surgical assistant operating on the patient. Despite its capability of performing abdominal procedures, the DAVINCI system is too bulky, and lacks the precision and dexterity required for delicate applications that require higher accuracy. Therefore, researchers are actively developing and implementing novel robotic systems to accommodate more demanding surgical procedures. Robot-assisted surgery presents many challenges, out of which nonlinear manipulation, high-precision dexterous operation, distal tool dexterity, insertion depth perception and contact force feedback are major concerns. Researchers have started to 
investigate some of these aforementioned concerns by developing robotic assistants, but a comprehensive robotic system that is capable of assisting general surgical procedures for eyes, heart and addressing existing surgical challenges is still missing. Besides, there are also many interesting robotics-related theoretical problems to be investigated under the light of surgery, e.g. multi-arm manipulation, robot performance evaluation, highprecision robot design, force sensing implementation, etc.

Design a robust controller for multi input-multi output (MIMO) nonlinear uncertain dynamical system (surgical robot manipulator) is the main challenging work in this research. Robot manipulators are set of links which connected by joints, they are multi input and multi output (MIMO), nonlinear, time variant, uncertain dynamic systems and are developed either to replace human work in many fields such as in industrial or in the manufacturing. Complexities of the tasks caused to design mechanical architectures and robot manipulator with nonlinear behavior. These factors are:

- Time-varying parameters based on tear and ware.

- Simplifying suppositions in system modeling caused to have un-modeled dynamic parameters.

- External disturbance and noise measurement which it is caused to generate uncertainties [6].

A controller (control system) is a device, which cans sense information from system to improvement the dynamic behavior of first order delay system based on actuation and computation. From scientific perspectives, control theory is divided into two parts; linear control theory and nonlinear control theory [6,1-3]. Linear control theory is divided into following groups:

- Proportional-Derivative (PD) control algorithm

- Proportional-Integral (PI) control algorithm

- Proportional-Integral-Derivative (PID) control algorithm

Nonlinear control theory is also has two main divisions;

- Conventional control theory

- Soft computing control theory

Conventional nonlinear control theories are highly sensitive to system's behavior and work based on cancelling decoupling and nonlinear terms of dynamic parameters of each likes in robot manipulators. Computed Torque Control (CTC) and Sliding Mode Control (SMC) are two nonlinear conventional controller which introduced by many researchers to control of types of robot manipulator [1-5].

Computed Torque Controller (CTC) is one of the effective nonlinear controllers to control of robot manipulator [6-7]. Consequently, to have a good performance, linearization and decoupling without using many gears, feedback linearization (computedtorque) control methodologies is presented. To design computed torque controller, an accurate dynamic model of robot manipulator plays an important role. To modelling an accurate dynamic system, modelling of complex parameters is needed to form the structure of system's dynamic model. It may be very difficult to include all the complexities in the system dynamic model [7]. In this research PD computed torque controller is designed for 4-DOF surgical robot to improve the sensitivity.

This paper is organized as follows; second part focuses on the system modeling dynamic formulation and Design linear controller. Third part is focused on the methodology, which focused on design model-reference based PD computed torque controller. Simulation result and discussion is illustrated in forth part. The last part focuses on the conclusion and compare between this method and the other ones.

\section{Theory}

System's Dynamic: A dynamic function is the study of motion with regard to the forces. Dynamic modeling of surgical robot manipulators is used to illustrate the behavior 
of robot manipulator (e.g., nonlinear dynamic behavior), design of nonlinear conventional controller and for simulation. It is used to analyses the relationship between dynamic functions output (e.g., joint motion, velocity, and accelerations) to input source of dynamic functions (e.g., force/torque or current/voltage). Dynamic functions is also used to explain the some dynamic parameter's effect (e.g., inertial matrix, Coriolios, Centrifugal, and some other parameters) to system's behavior [3].

The equation of multi degrees of freedom (DOF) surgical robot manipulator dynamics is considered by the following equation[7]:

$$
[\boldsymbol{A}(\boldsymbol{q})] \ddot{\boldsymbol{q}}+[\boldsymbol{N}(\boldsymbol{q}, \dot{\boldsymbol{q}})]=[\boldsymbol{\tau}]
$$

Where $\tau$ is actuator's torque and is $n \times 1$ vector, A (q) is positive define inertia and is $n \times n$ symmetric matrix based on the following formulation;

$$
A(q)=\left[\begin{array}{cccccc}
A_{11} & A_{12} & \ldots & \ldots & \ldots . & A_{1 n} \\
A_{21} & \ldots & \ldots & \ldots & \ldots . & A_{2 n} \\
\ldots & \ldots & \ldots & \ldots & \ldots & \ldots \\
\ldots & \ldots & \ldots & \ldots & \ldots & \ldots \\
\ldots & \ldots & \ldots & \ldots & \ldots & \ldots \\
A_{n .1} & \ldots & \ldots & \ldots & \ldots & A_{n . n}
\end{array}\right]
$$

, $N(q, \dot{q})$ is the vector of nonlinearity term, and q is $n \times 1$ joints variables. If all joints are revolute, the joint variables are angle $(\theta)$ and if these joints are translated, the joint variables are translating position $(d)$. The nonlinearity term of robot manipulator is derived as three main parts; Coriolis $\boldsymbol{b}(\boldsymbol{q})$, Centrifugal $\boldsymbol{C}(\boldsymbol{q})$, and Gravity $\boldsymbol{G}(\boldsymbol{q})$. Consequently the robot manipulator dynamic equation can also be written as [7]:

$$
\begin{gathered}
{[N(\boldsymbol{q}, \dot{\boldsymbol{q}})]=[V(\boldsymbol{q}, \dot{\boldsymbol{q}})]+[\boldsymbol{G}(\boldsymbol{q})]} \\
{[V(\boldsymbol{q}, \dot{\boldsymbol{q}})]=[\boldsymbol{b}(\boldsymbol{q})][\dot{\boldsymbol{q}} \dot{\boldsymbol{q}}]+[\boldsymbol{C}(\boldsymbol{q})][\dot{\boldsymbol{q}}]^{2}} \\
\tau=A(\boldsymbol{q}) \ddot{\boldsymbol{q}}+\boldsymbol{b}(\boldsymbol{q})[\dot{\boldsymbol{q}} \dot{\boldsymbol{q}}]+\boldsymbol{C}(\boldsymbol{q})[\dot{\boldsymbol{q}}]^{2}+\boldsymbol{G}(\boldsymbol{q})
\end{gathered}
$$

Where,

$b(q)$ is a Coriolis torque matrix and is $n \times \frac{n \times(n-1)}{2}$ matrix, $C(q)$ is Centrifugal torque matrix and is $n \times n$ matrix, Gravity is the force of gravity and is $n \times 1$ matrix, [ $\dot{q} \dot{q}]$ is vector of joint velocity that it can give by: $\left[\dot{q}_{1} . \dot{q}_{2}, \dot{q}_{1} . \dot{q}_{3}, \ldots ., \dot{q}_{1} . \dot{q}_{n}, \dot{q}_{2} . \dot{q}_{3}, \ldots . .\right]^{T}$, and $[\dot{q}]^{2}$ is vector, that it can given by: $\left[\dot{q}_{1}{ }^{2}, \dot{q}_{2}{ }^{2}, \dot{q}_{3}{ }^{2}, \ldots .\right]^{T}$. According to the basic information from system's modelling, all functions are derived as the following form;

$$
\text { Outputs }=\text { function (inputs) }
$$

In the dynamic formulation of robot manipulator the inputs are torques matrix and the outputs are actual joint variables,

$$
\begin{gathered}
q=\text { function }(\tau) \\
\ddot{\boldsymbol{q}}=\boldsymbol{A}^{-1}(\boldsymbol{q}) \cdot\{\boldsymbol{\tau}-\boldsymbol{N}(\boldsymbol{q}, \dot{\boldsymbol{q}})\} \\
q=\iint \boldsymbol{A}^{-1}(\boldsymbol{q}) \cdot\{\boldsymbol{\tau}-\boldsymbol{N}(\boldsymbol{q}, \dot{\boldsymbol{q}})\}
\end{gathered}
$$

The Coriolis matrix $(b)$ is a $n \times \frac{n(n-1)}{2}$ matrix which calculated as follows; 


$$
\boldsymbol{b}(\boldsymbol{q})=\left[\begin{array}{cccccccccc}
\boldsymbol{b}_{112} & \boldsymbol{b}_{113} & \ldots & \boldsymbol{b}_{11 n} & \boldsymbol{b}_{123} & \ldots & \boldsymbol{b}_{12 n} & \ldots & \ldots & \boldsymbol{b}_{1 . n-1 . n} \\
\boldsymbol{b}_{212} & \ldots & \ldots & \boldsymbol{b}_{21 n} & \boldsymbol{b}_{223} & \ldots & \ldots & \ldots & \ldots & \boldsymbol{b}_{2 . n-1 . n} \\
\ldots & \ldots & \ldots & \ldots & \ldots & \ldots & \ldots & \ldots & \ldots & \ldots \\
\ldots & \ldots & \ldots & \ldots & \ldots & \ldots & \ldots & \ldots & \ldots & \ldots \\
\ldots & \ldots & \ldots & \ldots & \ldots & \ldots & \ldots & \ldots & \ldots & \ldots \\
\boldsymbol{b}_{n .1 .2} & \ldots & \ldots & \boldsymbol{b}_{n .1 . n} & \ldots & \ldots & \ldots & \ldots & \ldots & \boldsymbol{b}_{n . n-1 . n}
\end{array}\right]
$$

The Centrifugal matrix $(\mathrm{C})$ is a $n \times n$ matrix;

$$
C(q)=\left[\begin{array}{ccc}
C_{11} & \cdots & C_{1 n} \\
\vdots & \ddots & \vdots \\
C_{n 1} & \cdots & C_{n n}
\end{array}\right]
$$

The Gravity vector $(\mathrm{G})$ is a $n \times 1$ vector;

$$
G(q)=\left[\begin{array}{c}
g_{1} \\
g_{2} \\
\vdots \\
g_{n}
\end{array}\right]
$$

The dynamic formulations for 4 Degrees of Freedom serial links surgical robot manipulator are computed by;

Where

$$
A(\ddot{\theta})\left[\begin{array}{c}
\ddot{\theta}_{1} \\
\ddot{\theta}_{2} \\
\ddot{\theta}_{3} \\
\ddot{\theta}_{4}
\end{array}\right]+B(\theta)\left[\begin{array}{c}
\dot{\theta}_{1} \dot{\theta}_{2} \\
\dot{\theta}_{1} \dot{\theta}_{3} \\
\dot{\theta}_{1} \dot{\theta}_{4} \\
\dot{\theta}_{2} \dot{\theta}_{3} \\
\dot{\theta}_{2} \dot{\theta}_{4} \\
\dot{\theta}_{3} \dot{\theta}_{4}
\end{array}\right]+C(\theta)\left[\begin{array}{c}
\dot{\theta}_{1}^{2} \\
\dot{\theta}_{2}^{2} \\
\dot{\theta}_{3}^{2} \\
\dot{\theta}_{4}^{2}
\end{array}\right]+G(\theta)=\left[\begin{array}{c}
\tau_{1} \\
\tau_{2} \\
\tau_{3} \\
\tau_{4}
\end{array}\right]
$$

$$
A(q)=\left[\begin{array}{cccc}
A_{11} & A_{12} & A_{13} & 0 \\
A_{21} & A_{22} & A_{23} & 0 \\
A_{31} & A_{32} & A_{33} & 0 \\
0 & 0 & 0 & A_{44}
\end{array}\right]
$$

According to [7] the inertial matrix elements $(\boldsymbol{A})$ are

$$
\begin{aligned}
& A_{11}=I_{m 1}+I_{1}+I_{3} \times \cos \left(\theta_{2}\right) \cos \left(\theta_{2}\right)+I_{7} \sin \left(\theta_{2}+\theta_{3}\right) \sin \left(\theta_{2}+\theta_{3}\right) \\
& +I_{10} \sin \left(\theta_{2}+\theta_{3}\right) \cos \left(\theta_{2}+\theta_{3}\right)+I_{11} \sin \left(\theta_{2}\right) \cos \left(\theta_{2}\right) \\
& +I_{21} \sin \left(\theta_{2}+\theta_{3}\right) \sin \left(\theta_{2}+\theta_{3}\right)+2 \\
& +\left[I_{5} \cos \left(\theta_{2}\right) \sin \left(\theta_{2}+\theta_{3}\right)+I_{12} \cos \left(\theta_{2}\right) \cos \left(\theta_{2}+\theta_{3}\right)\right. \\
& +I_{15} \sin \left(\theta_{2}+\theta_{3}\right) \sin \left(\theta_{2}+\theta_{3}\right)+I_{16} \cos \left(\theta_{2}\right) \sin \left(\theta_{2}+\theta_{3}\right) \\
& +I_{22} \sin \left(\theta_{2}+\theta_{3}\right) \cos \left(\theta_{2}+\theta_{3}\right) \\
& A_{12}=I_{4} \sin \left(\theta_{2}\right)+I_{8} \cos \left(\theta_{2}+\theta_{3}\right)+I_{9} \cos \left(\theta_{2}\right)+I_{13} \sin \left(\theta_{2}+\theta_{3}\right) \\
& -I_{18} \cos \left(\theta_{2}+\theta_{3}\right) \\
& A_{13}=I_{8} \cos \left(\theta_{2}+\theta_{3}\right)+I_{13} \sin \left(\theta_{2}+\theta_{3}\right)-I_{18} \cos \left(\theta_{2}+\theta_{3}\right) \\
& A_{22}=I_{m 2}+I_{2}+I_{6}+2\left[I_{5} \sin \left(\theta_{3}\right)+I_{12} \cos \left(\theta_{2}\right)+I_{15}+I_{16} \sin \left(\theta_{3}\right)\right. \\
& A_{23}=I_{5} \sin \left(\theta_{3}\right)+I_{6}+I_{12} \cos \left(\theta_{3}\right)+I_{16} \sin \left(\theta_{3}\right)+2 I_{15} \\
& A_{33}=I_{m 3}+I_{6}+2 I_{15}
\end{aligned}
$$




$$
\begin{gathered}
A_{44}=I_{m 4}+I_{14} \\
A_{21}=A_{12}, A_{31}=A_{13} \text { and } A_{32}=A_{23}
\end{gathered}
$$

The Corilios $(\boldsymbol{b})$ matrix elements are;

$$
b(q)=\left[\begin{array}{cccc}
b_{112} & b_{113} & 0 & b_{123} \\
0 & 0 & b_{214} & b_{223} \\
0 & 0 & b_{314} & 0 \\
b_{412} & b_{413} & 0 & 0
\end{array}\right]
$$

Where,

$$
\begin{aligned}
& b_{112}=2\left[-I_{3} \sin \left(\theta_{2}\right) \cos \left(\theta_{2}\right)+I_{5} \cos \left(\theta_{2}+\theta_{2}+\theta_{3}\right)\right. \\
& +I_{7} \sin \left(\theta_{2}+\theta_{3}\right) \cos \left(\theta_{2}+\theta_{3}\right)-I_{12} \sin \left(\theta_{2}+\theta_{2}+\theta_{3}\right) \\
& -I_{15} 2 \sin \left(\theta_{2}+\theta_{3}\right) \cos \left(\theta_{2}+\theta_{3}\right)+I_{16} \cos \left(\theta_{2}+\theta_{2}+\theta_{3}\right) \\
& +I_{21} \sin \left(\theta_{2}+\theta_{3}\right) \cos \left(\theta_{2}+\theta_{3}\right) \\
& \left.+I_{22}\left(1-2 \sin \left(\theta_{2}+\theta_{3}\right) \sin \left(\theta_{2}+\theta_{3}\right)\right)\right] \\
& +I_{10}\left(1-2 \sin \left(\theta_{2}+\theta_{3}\right) \sin \left(\theta_{2}+\theta_{3}\right)\right) \\
& +I_{11}\left(1-2 \sin \left(\theta_{2}\right) \sin \left(\theta_{2}\right)\right) \\
& b_{113}=2\left[I_{5} \cos \left(\theta_{2}\right) \cos \left(\theta_{2}+\theta_{3}\right)+I_{7} \sin \left(\theta_{2}+\theta_{3}\right) \cos \left(\theta_{2}+\theta_{3}\right)\right. \\
& -I_{12} \cos \left(\theta_{2}\right) \sin \left(\theta_{2}+\theta_{2}\right) \\
& +I_{15} 2 \sin \left(\theta_{2}+\theta_{3}\right) \cos \left(\theta_{2}+\theta_{3}\right)+I_{16} \cos \left(\theta_{2}\right) \cos \left(\theta_{2}+\theta_{3}\right) \\
& +I_{21} \sin \left(\theta_{2}+\theta_{3}\right) \cos \left(\theta_{2}+\theta_{3}\right) \\
& \left.+I_{22}\left(1-2 \sin \left(\theta_{2}+\theta_{3}\right) \sin \left(\theta_{2}+\theta_{3}\right)\right)\right] \\
& +I_{10}\left(1-2 \sin \left(\theta_{2}+\theta_{3}\right) \sin \left(\theta_{2}+\theta_{3}\right)\right) \\
& b_{123}=2\left[-I_{8} \sin \left(\theta_{2}+\theta_{3}\right)+I_{13} \cos \left(\theta_{2}+\theta_{3}\right)+I_{18} \sin \left(\theta_{2}+\theta_{3}\right)\right] \\
& b_{214}=I_{14} \sin \left(\theta_{2}+\theta_{3}\right)+I_{19} \sin \left(\theta_{2}+\theta_{3}\right)+2 I_{20} \sin \left(\theta_{2}+\theta_{3}\right)(1-0.5) \\
& b_{223}=2\left[-I_{12} \sin \left(\theta_{3}\right)+I_{5} \cos \left(\theta_{3}\right)+I_{16} \cos \left(\theta_{3}\right)\right] \\
& b_{314}=2\left[I_{20} \sin \left(\theta_{2}+\theta_{3}\right)(1-0.5)\right]+I_{14} \sin \left(\theta_{2}+\theta_{3}\right)+I_{19} \sin \left(\theta_{2}+\theta_{3}\right) \\
& b_{412}=b_{214}=-\left[I_{14} \sin \left(\theta_{2}+\theta_{3}\right)+I_{19} \sin \left(\theta_{2}+\theta_{3}\right)\right. \\
& \left.+2 I_{20} \sin \left(\theta_{2}+\theta_{3}\right)(1-0.5)\right] \\
& b_{413}=-b_{314}=-2\left[I_{20} \sin \left(\theta_{2}+\theta_{3}\right)(1-0.5)\right]+I_{14} \sin \left(\theta_{2}+\theta_{3}\right) \\
& +I_{19} \sin \left(\theta_{2}+\theta_{3}\right)
\end{aligned}
$$

Based on above discussion $[\boldsymbol{b}(\boldsymbol{q})]$ is $4 \times 6$ matrix and $[\dot{\boldsymbol{q}} \dot{\boldsymbol{q}}]$ is $6 \times 1$, therefore $[\boldsymbol{b}(\boldsymbol{q}) \cdot \dot{\boldsymbol{q}} \dot{\boldsymbol{q}}]$ is $4 \times 1$.

$$
[b(q) \cdot \dot{q} \dot{q}]_{4 \times 1}=\left[\begin{array}{c}
b_{112} \cdot q_{1} q_{2}+b_{113} \cdot q_{1} q_{3}+0+b_{123} \cdot q_{2} q_{3} \\
0+b_{214} \cdot q_{1} q_{4}+b_{223} \cdot q_{2} q_{3} \\
b_{314} \cdot q_{1} q_{4} \\
b_{412} \cdot q_{1} q_{2}+b_{413} \cdot q_{1} q_{3}
\end{array}\right]
$$

According to [7] Centrifugal $(\boldsymbol{C})$ matrix elements are;

$$
C(q)=\left[\begin{array}{cccc}
0 & C_{12} & C_{13} & 0 \\
C_{21} & C_{22} & C_{23} & 0 \\
C_{31} & C_{32} & 0 & 0 \\
0 & 0 & 0 & 0
\end{array}\right]
$$


Where,

$$
\begin{aligned}
& c_{12}=I_{4} \cos \left(\theta_{2}\right)-I_{8} \sin \left(\theta_{2}+\theta_{3}\right)-I_{9} \sin \left(\theta_{2}\right)+I_{13} \cos \left(\theta_{2}+\theta_{3}\right) \\
& +I_{18} \sin \left(\theta_{2}+\theta_{3}\right) \\
& c_{13}=0.5 b_{123}=-I_{8} \sin \left(\theta_{2}+\theta_{3}\right)+I_{13} \cos \left(\theta_{2}+\theta_{3}\right)+I_{18} \sin \left(\theta_{2}+\theta_{3}\right) \\
& c_{21}=-0.5 b_{112} \\
& =I_{3} \sin \left(\theta_{2}\right) \cos \left(\theta_{2}\right)-I_{5} \cos \left(\theta_{2}+\theta_{2}+\theta_{3}\right) \\
& -I_{7} \sin \left(\theta_{2}+\theta_{3}\right) \cos \left(\theta_{2}+\theta_{3}\right)+I_{12} \sin \left(\theta_{2}+\theta_{2}+\theta_{3}\right) \\
& +I_{15} 2 \sin \left(\theta_{2}+\theta_{3}\right) \cos \left(\theta_{2}+\theta_{3}\right)-I_{16} \cos \left(\theta_{2}+\theta_{2}+\theta_{3}\right) \\
& -I_{21} \sin \left(\theta_{2}+\theta_{3}\right) \cos \left(\theta_{2}+\theta_{3}\right) \\
& -I_{22}\left(1-2 \sin \left(\theta_{2}+\theta_{3}\right) \sin \left(\theta_{2}+\theta_{3}\right)\right) \\
& -0.5 I_{10}\left(1-2 \sin \left(\theta_{2}+\theta_{3}\right) \sin \left(\theta_{2}+\theta_{3}\right)\right) \\
& -0.5 I_{11}\left(1-2 \sin \left(\theta_{2}\right) \sin \left(\theta_{2}\right)\right) \\
& c_{22}=0.5 b_{223}=-I_{12} \sin \left(\theta_{3}\right)+I_{5} \cos \left(\theta_{3}\right)+I_{16} \cos \left(\theta_{3}\right) \\
& c_{23}=-0.5 b_{113} \\
& =-I_{5} \cos \left(\theta_{2}\right) \cos \left(\theta_{2}+\theta_{3}\right)-I_{7} \sin \left(\theta_{2}+\theta_{3}\right) \cos \left(\theta_{2}+\theta_{3}\right) \\
& +I_{12} \cos \left(\theta_{2}\right) \sin \left(\theta_{2}+\theta_{2}\right)-I_{15} 2 \sin \left(\theta_{2}+\theta_{3}\right) \cos \left(\theta_{2}+\theta_{3}\right) \\
& -I_{16} \cos \left(\theta_{2}\right) \cos \left(\theta_{2}+\theta_{3}\right)-I_{21} \sin \left(\theta_{2}+\theta_{3}\right) \cos \left(\theta_{2}+\theta_{3}\right) \\
& -I_{22}\left(1-2 \sin \left(\theta_{2}+\theta_{3}\right) \sin \left(\theta_{2}+\theta_{3}\right)\right) \\
& -0.5 I_{10}\left(1-2 \sin \left(\theta_{2}+\theta_{3}\right) \sin \left(\theta_{2}+\theta_{3}\right)\right) \\
& c_{31}=-c_{23}=I_{12} \sin \left(\theta_{3}\right)-I_{5} \cos \left(\theta_{3}\right)-I_{16} \cos \left(\theta_{3}\right) \\
& c_{32}=\sin \left(\theta_{2}+\theta_{3}\right) \cos \left(\theta_{2}+\theta_{3}\right)-I_{15} 2 \sin \left(\theta_{2}+\theta_{3}\right) \cos \left(\theta_{2}+\theta_{3}\right) \\
& -I_{16} \cos \left(\theta_{2}\right) \cos \left(\theta_{2}+\theta_{3}\right)-I_{22} \cos \left(\theta_{2}+\theta_{3}\right) \cos \left(\theta_{2}+\theta_{3}\right) \\
& {\left[C(q) \cdot \dot{q}^{2}\right]_{6 \times 1}=\left[\begin{array}{l}
c_{12} \cdot q_{2}^{2}+c_{13} \cdot q_{3}^{2} \\
c_{21} \cdot q_{1}^{2}+c_{23} \cdot q_{3}^{2} \\
c_{13} \cdot q_{1}^{2}+c_{32} \cdot q_{2}^{2} \\
0
\end{array}\right]}
\end{aligned}
$$

Gravity $(\boldsymbol{G})$ Matrix elements are;

$$
[G(q)]_{4 \times 1}=\left[\begin{array}{c}
0 \\
G_{2} \\
G_{3} \\
0
\end{array}\right]
$$

Where,

$$
\begin{gathered}
G_{2}=g_{1} \cos \left(\theta_{2}\right)+g_{2} \sin \left(\theta_{2}+\theta_{3}\right)+g_{3} \sin \left(\theta_{2}\right)+g_{4} \cos \left(\theta_{2}+\theta_{3}\right) \\
+g_{5} \sin \left(\theta_{2}+\theta_{3}\right) \\
G_{3}=g_{2} \sin \left(\theta_{2}+\theta_{3}\right)+g_{4} \cos \left(\theta_{2}+\theta_{3}\right)+g_{5} \sin \left(\theta_{2}+\theta_{3}\right)
\end{gathered}
$$

$$
\text { If }[I]_{4 \times 1}=[B]_{4 \times 1}+[C]_{4 \times 1}+[G]_{4 \times 1}
$$

Then $\ddot{q}$ is written as follows;

$$
[\ddot{q}]_{4 \times 1}=\left[A^{-1}(q)\right]_{4 \times 4} \times\left\{[\tau]_{4 \times 1}-[I]_{4 \times 1}\right\}
$$


$K$ is presented as follows;

$$
\begin{gathered}
{[K]_{4 \times 1}=\left\{[\tau]_{4 \times 1}-[I]_{4 \times 1}\right\}} \\
{[\ddot{q}]_{4 \times 1}=\left[A^{-1}(q)\right]_{4 \times 4} \times[K]_{4 \times 1}} \\
{[q]_{4 \times 1}=\iint\left[A^{-1}(q)\right]_{4 \times 4} \times[K]_{4 \times 1}}
\end{gathered}
$$

Basic information about inertial and gravitational constants is show in Tables 1 and 2

Table 1. Inertial Constant Reference (Kg. $\left.M^{2}\right)$

\begin{tabular}{|c|c|}
\hline$I_{1}=1.43 \pm 0.05$ & $I_{2}=1.75 \pm 0.07$ \\
\hline$I_{3}=1.38 \pm 0.05$ & $I_{4}=0.69 \pm 0.02$ \\
\hline$I_{5}=0.372 \pm 0.031$ & $I_{6}=0.333 \pm 0.016$ \\
\hline$I_{7}=0.298 \pm 0.029$ & $I_{8}=-0.134 \pm 0.014$ \\
\hline$I_{9}=0.0238 \pm 0.012$ & $I_{10}=-0.0213 \pm 0.0022$ \\
\hline$I_{11}=-0.0142 \pm 0.0070$ & $I_{12}=-0.011 \pm 0.0011$ \\
\hline$I_{13}=-0.00379 \pm 0.0009$ & $I_{14}=0.00164 \pm 0.000070$ \\
\hline$I_{15}=0.00125 \pm 0.0003$ & $I_{16}=0.00124 \pm 0.0003$ \\
\hline$I_{17}=0.000642 \pm 0.0003$ & $I_{18}=0.000431 \pm 0.00013$ \\
\hline$I_{19}=0.0003 \pm 0.0014$ & $I_{20}=-0.000202 \pm 0.0008$ \\
\hline$I_{21}=-0.0001 \pm 0.0006$ & $I_{22}=-0.000058 \pm 0.000015$ \\
\hline$I_{23}=0.00004 \pm 0.00002$ & $I_{m 1}=1.14 \pm 0.27$ \\
\hline$I_{m 2}=4.71 \pm 0.54$ & $I_{m 3}=0.827 \pm 0.093$ \\
\hline$I_{m 4}=0.2 \pm 0.016$ & $I_{m 5}=0.179 \pm 0.014$ \\
\hline$I_{m 6}=0.193 \pm 0.016$ & \\
\hline
\end{tabular}

Table 2. Gravitational Constant (N.m)

\begin{tabular}{|c|c|}
\hline$g_{1}=-37.2 \pm 0.5$ & $g_{2}=-8.44 \pm 0.20$ \\
\hline$g_{3}=1.02 \pm 0.50$ & $g_{4}=0.249 \pm 0.025$ \\
\hline$g_{5}=-0.0282 \pm 0.0056$ & \\
\hline
\end{tabular}

Figure 1. Shows The Simscape Modeling of Robot. 


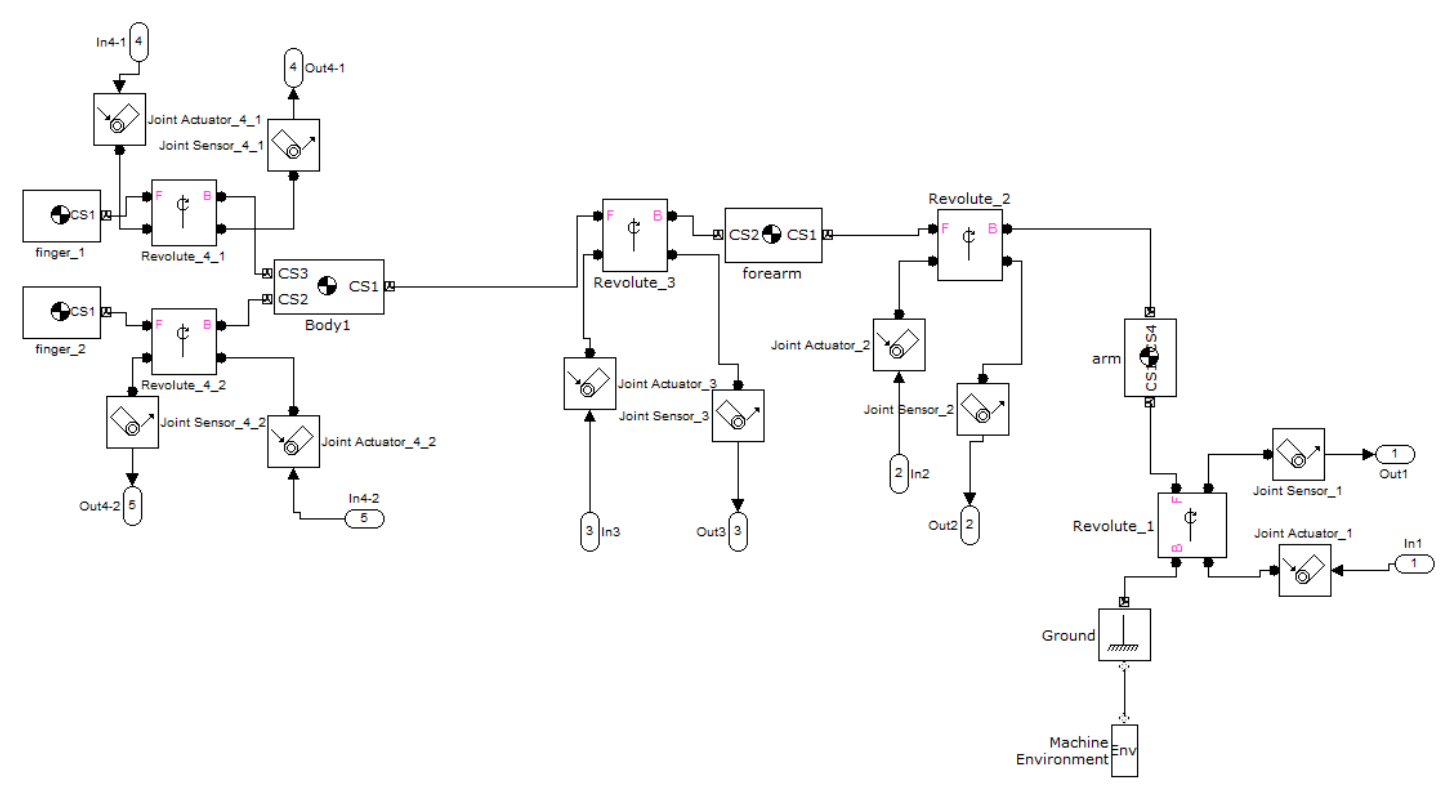

Figure 1. System's Model

System's physical model shows as follow:

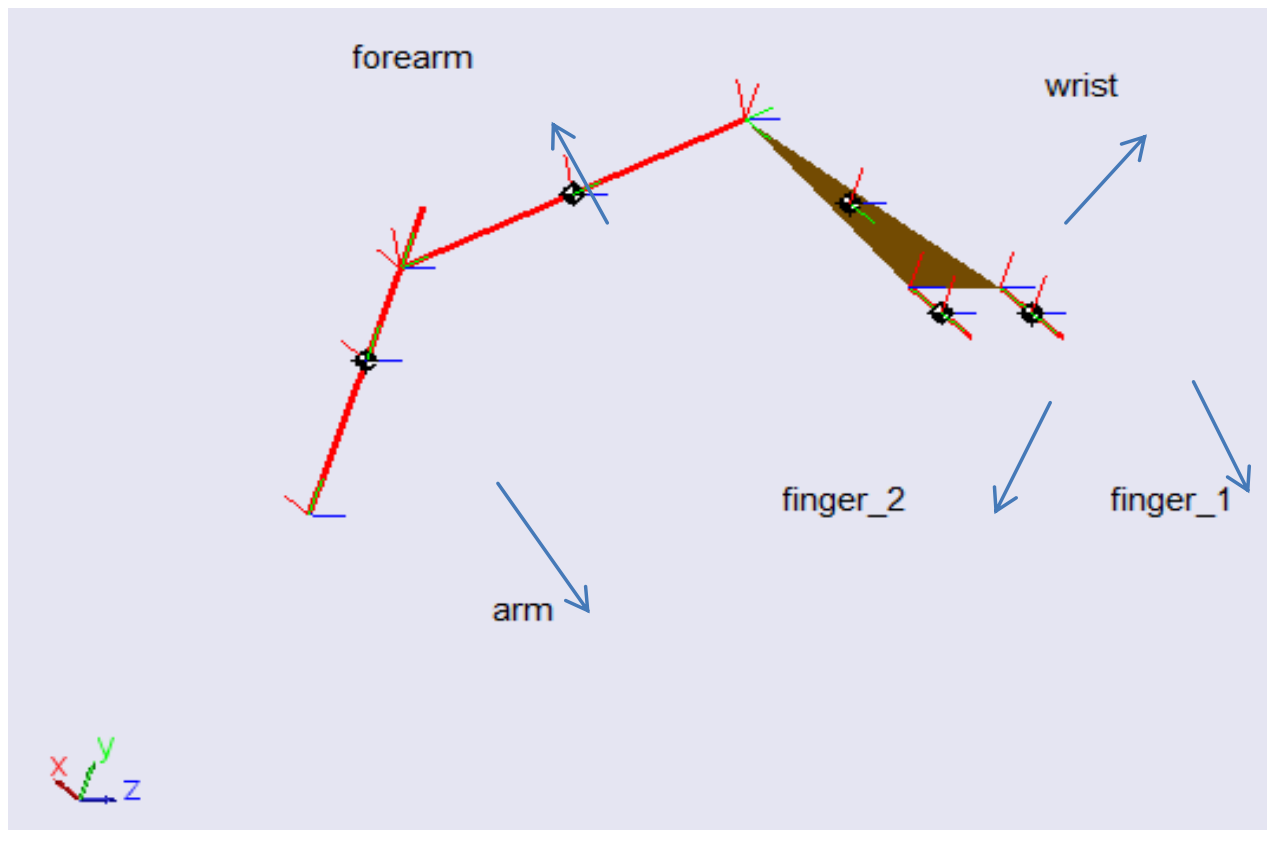

Figure 2. System's Physical Model

Proportional plus Derivative (PD) control: This type of linear controller is widely used in control process where the results are sensitive to exceeded of set point. This controller, like Proportional controller, has permanent variation in presence of selflimitation control. In mathematically, the formulation of Proportional-Derivative part calculated as follows;

$$
U_{P D}=K_{p} \times e+K_{v}\left(\frac{d e}{d t}\right)=K_{p} \times e+K_{v} \dot{e}
$$


The Derivative component in this type of methodology is used to cancel outs the change process variables change in presence of quick change in controllers input. Figure 3 shows the block diagram of Proportional-Derivative (PD) controller.

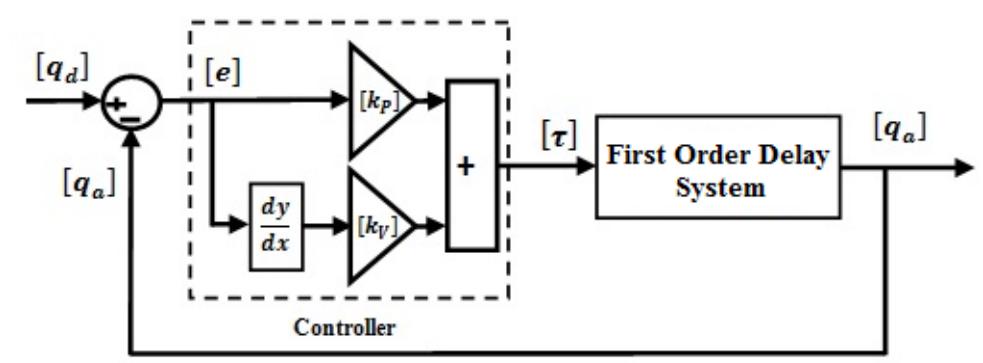

Figure 3. Block Diagram of PD Controller

Figure 4 shows the ramp response of PD controller.

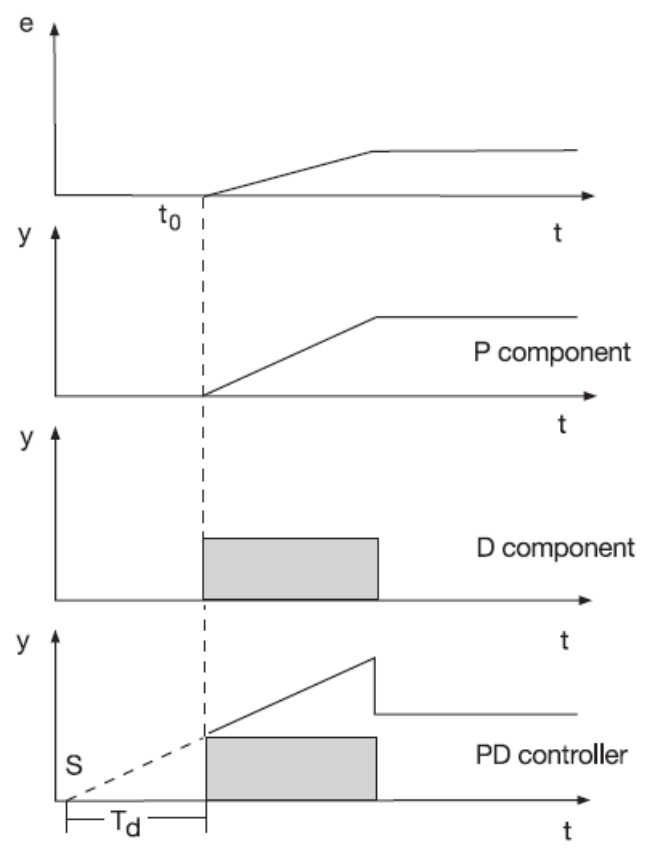

Figure 4. Ramp Response of a PD Controller [13]

Proportional plus Integral (PI) control: According to integral type of controller, it takes relatively long time. The proportional type controller used to immediately response to the input variations. The proportional-integral (PI) controller has the advantages of both proportional and integral controller; it is rapid response to the input deviation as well as the exact control at the desired input. Figure 5 shows the block diagram of PI control of FOD system.

$$
U_{P I}=K_{p} \times e+K_{i}\left(\frac{1}{T} \int e . d t\right)=K_{p} \times e+K_{i} \sum e
$$




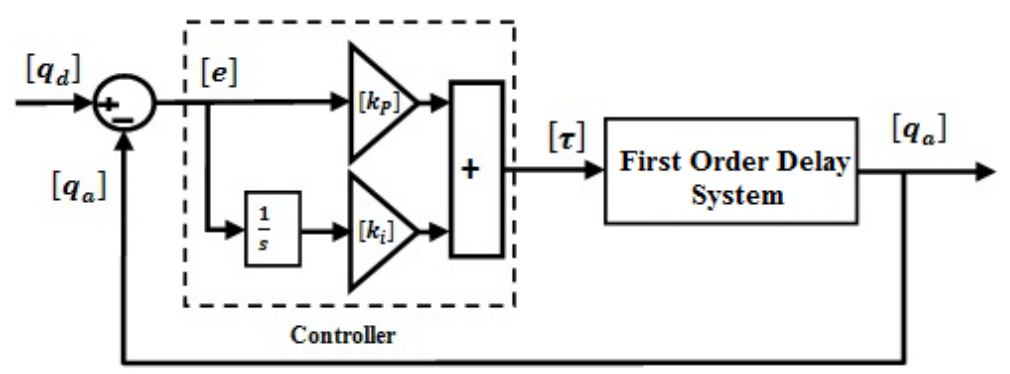

Figure 5. Block Diagram of PI Controller for First Order Delay System

Figure 6 shows the step response of PI controller.

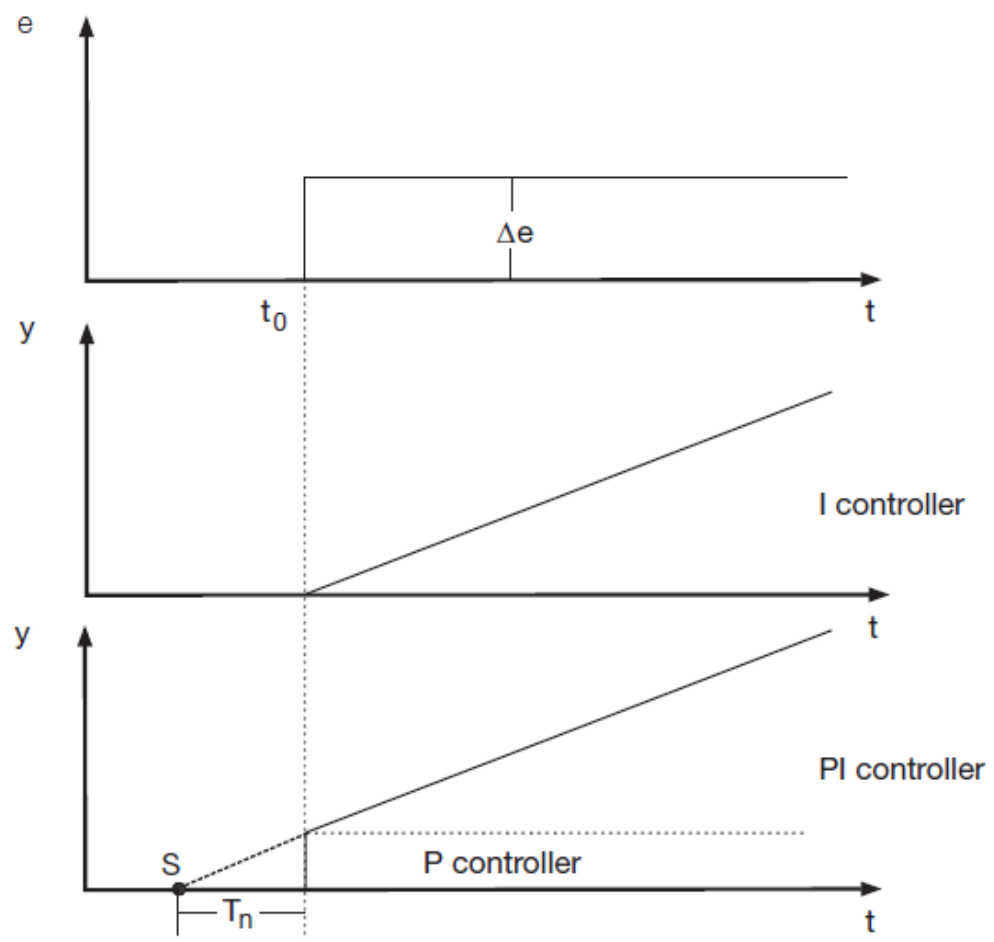

Figure 6. Step Response of a Proportional-Integral (PI) Controller [13]

Proportional-Integral-Derivative (PID) control: This type of controller has rapid response to the input deviation, the exact control at the desired input as well as fast response to the disturbances. The PID controller takes the error between the desired joint variables and the actual joint variables to control the serial links robot manipulator. The equation of PID controller for control of 4 degrees of freedom serial links PUMA robot manipulator is;

$$
\left[\begin{array}{l}
\widehat{\tau_{1}} \\
\widehat{\tau_{2}} \\
\widehat{\tau_{3}} \\
\widehat{\tau_{4}}
\end{array}\right]=\left[\begin{array}{l}
K_{i 1} \sum e_{1}+K_{v 1} \dot{e}_{1}+K_{p 1} e_{1} \\
K_{i 2} \sum e_{2}+K_{v 2} \dot{e}_{2}+K_{p 2} e_{2} \\
K_{i 3} \sum e_{3}+K_{v 3} \dot{e}_{3}+K_{p 3} e_{3} \\
K_{i 4} \sum e_{4}+K_{v 4} \dot{e}_{4}+K_{p 4} e_{4}
\end{array}\right]
$$

Where $e=q_{d}-q_{a}, q_{d}$ is desired joint variable and $q_{a}$ is actual joint variable. 
Figure 7 shows the block diagram of PID control of robot manipulator.

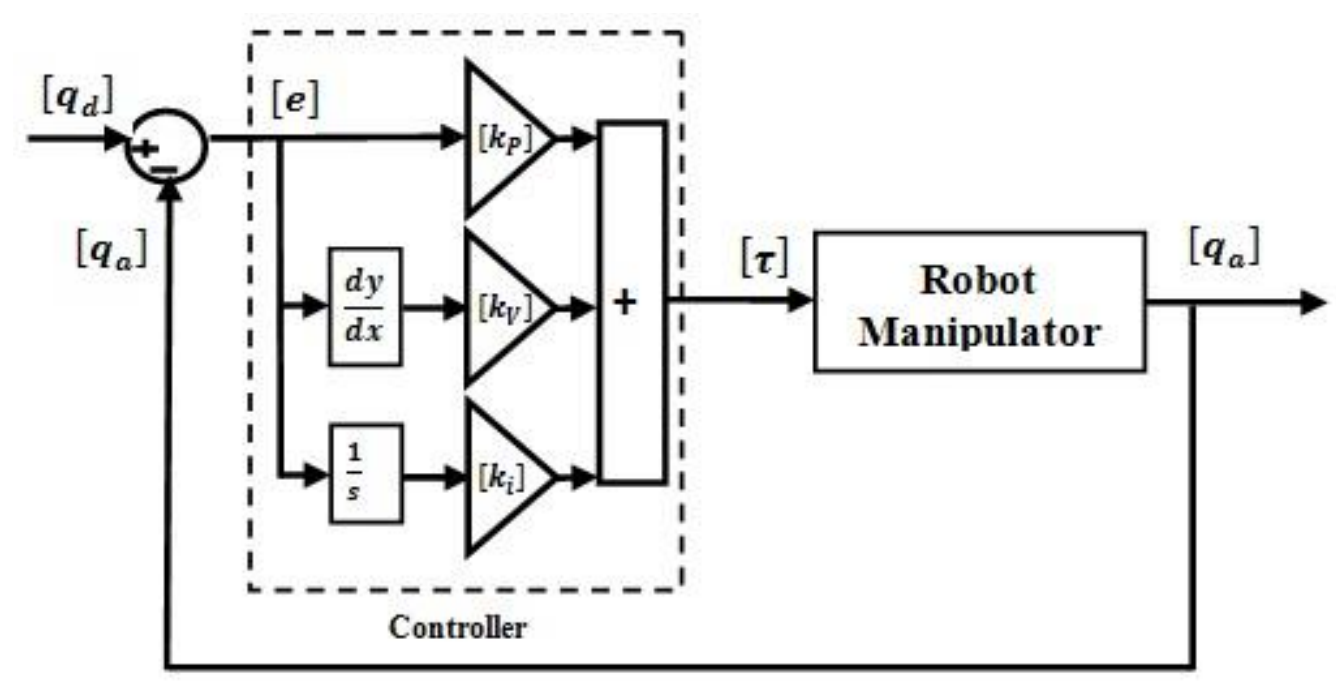

Figure 7. Block Diagram of PID Control of Robot Manipulator

Stability Proof of Linear Controller: In PD controller the control law is given by the following equation;

$$
\tau=K_{p_{i}} e+K_{v_{i}} \dot{e}
$$

Where $\boldsymbol{e}=\boldsymbol{q}_{\boldsymbol{i}_{\boldsymbol{d}}}-\boldsymbol{q}_{\boldsymbol{i}_{\boldsymbol{a}}}$ and $\dot{\boldsymbol{e}}=\dot{\boldsymbol{q}}_{\boldsymbol{i}_{\boldsymbol{d}}}-\dot{\boldsymbol{q}}_{\boldsymbol{i}_{\boldsymbol{a}}}$.

In this theory $\boldsymbol{K}_{\boldsymbol{p}_{\boldsymbol{i}}}$ and $\boldsymbol{K}_{\boldsymbol{v}_{\boldsymbol{i}}}$ are positive constant. To show this controller is stable and achieves zero steady state error, the Lyapunov function is introduced;

$$
\begin{aligned}
V= & \frac{1}{2}\left[\dot{q}^{T} A(q) \dot{q}+e^{T} K_{p} e\right]= \\
& \frac{1}{2} \frac{d}{d t}\left[\dot{q}^{T} A \dot{q}\right]=\dot{q} \tau
\end{aligned}
$$

If the conversation energy is written by the following form:

$\overline{2} \frac{1}{d t}\left[\dot{q}^{T} A \dot{q}\right]=\dot{q} \tau$

Where $(\dot{\boldsymbol{q}} \boldsymbol{\tau})$ shows the power inputs from actuator and $\frac{1}{2} \frac{d}{d t}\left[\dot{q}^{T} A \dot{q}\right]$ is the derivative of the robot kinematic energy.

$$
\dot{V}=\dot{q}^{T}\left[\tau+K_{p} e\right]
$$

Based on $\boldsymbol{\tau}=-\boldsymbol{K}_{\boldsymbol{p}_{\boldsymbol{i}}} \boldsymbol{e}-\boldsymbol{K}_{\boldsymbol{v}_{\boldsymbol{i}}} \dot{\boldsymbol{e}}$, we can write

$$
\dot{V}=\dot{q}^{T} K_{p} \dot{q} \leq 0
$$

If $\dot{\boldsymbol{V}}=\mathbf{0}$, we have

$$
\dot{q}=0 \rightarrow \ddot{q}=0 \rightarrow \ddot{q}=A^{-1} K_{p} e \rightarrow e=0
$$

In this state, the actual trajectories converge to the desired state.

\section{Methodology}

Computed torque controller (CTC) is one of the effective nonlinear control methodologies for second order nonlinear system (e.g., robot manipulator). Computed torque controller works based on behavior (dynamic formulation) of robot manipulator 
which caused to works very well when all dynamic and physical parameters are known. In uncertain dynamic parameters when the robot manipulator has variation computed torque controller has challenges. In this type of controller, if the desired position trajectory for the manipulator defined as $\boldsymbol{q}_{\boldsymbol{d}}(\boldsymbol{t})$, and the actual position trajectory defined as $\boldsymbol{q}_{\boldsymbol{a}}(\boldsymbol{t})$, the tracking error calculated by;

$$
e(t)=q_{d}(t)-q_{a}(t)
$$

Based on this research the errors for 4 links are;

If state space equation is defined by;

$$
\left[\begin{array}{l}
e_{1} \\
e_{2} \\
e_{3} \\
e_{4}
\end{array}\right]=\left[\begin{array}{l}
q_{d_{1}}(t)-q_{a_{1}}(t) \\
q_{d_{2}}(t)-q_{a_{2}}(t) \\
q_{d_{3}}(t)-q_{a_{3}}(t) \\
q_{d_{4}}(t)-q_{a_{4}}(t)
\end{array}\right]
$$

$$
\dot{x}=A x+B U
$$

According to the Brunousky canonical form $\boldsymbol{U}$ is the nonlinearity term and defined by;

$$
U=\ddot{\boldsymbol{q}}_{\boldsymbol{d}}+\boldsymbol{A}^{-1}(\boldsymbol{q}) \cdot\{\boldsymbol{N}(\boldsymbol{q} \cdot \dot{\boldsymbol{q}})-\boldsymbol{\tau}\}
$$

Based on (59) and (60)

$$
\dot{x}=\left[\begin{array}{ll}
\mathbf{0} & I \\
0 & 0
\end{array}\right] x+\left[\begin{array}{l}
0 \\
I
\end{array}\right] U
$$

According to $x=\left[e^{T} \dot{e}^{T}\right]^{T}$

$$
\frac{d}{d t}\left[\begin{array}{l}
e \\
\dot{e}
\end{array}\right]=\left[\begin{array}{ll}
0 & I \\
0 & 0
\end{array}\right] \cdot\left[\begin{array}{l}
e \\
\dot{e}
\end{array}\right]+\left[\begin{array}{l}
0 \\
I
\end{array}\right] U
$$

Based on (60), the requirement torque calculated by;

$$
\tau=A(q)\left(\ddot{q}_{d}+U\right)+N(\dot{q}, q)
$$

According to nonlinear feed-back control low, (61) is nonlinear food-back controller and it is used to tracking of desired trajectory. According to the literature [7], $\mathrm{U}$ is the linear part of this nonlinear controller and the PD, PI or PID terms can be selected. The formulation of PD computed torque controller is;

$$
\tau=A(q)\left(\ddot{q}_{d}+K_{v} \dot{e}+K_{p} e\right)+N(q, \dot{q})
$$

The PI computed torque controller formulation is;

$$
\tau=A(q)\left(\ddot{q}_{d}+K_{p} e+K_{i} \sum e\right)+N(q, \dot{q})
$$

The PID computed torque controller formulation is;

$$
\tau=A(q)\left(\ddot{q}_{d}+K_{p} e+K_{v} \dot{e}+K_{i} \sum e\right)+N(q, \dot{q})
$$

Where $\boldsymbol{K}_{\boldsymbol{p}}, \boldsymbol{K}_{\boldsymbol{v}}$ and $\boldsymbol{K}_{\boldsymbol{i}}$ are the controller gain coefficients. This type of controller has two main important parts; partly linear part and nonlinear term of dynamic equivalent part.

Block diagram of Proportional-Derivative (PD) computed torque controller shows in Figure 8 . 


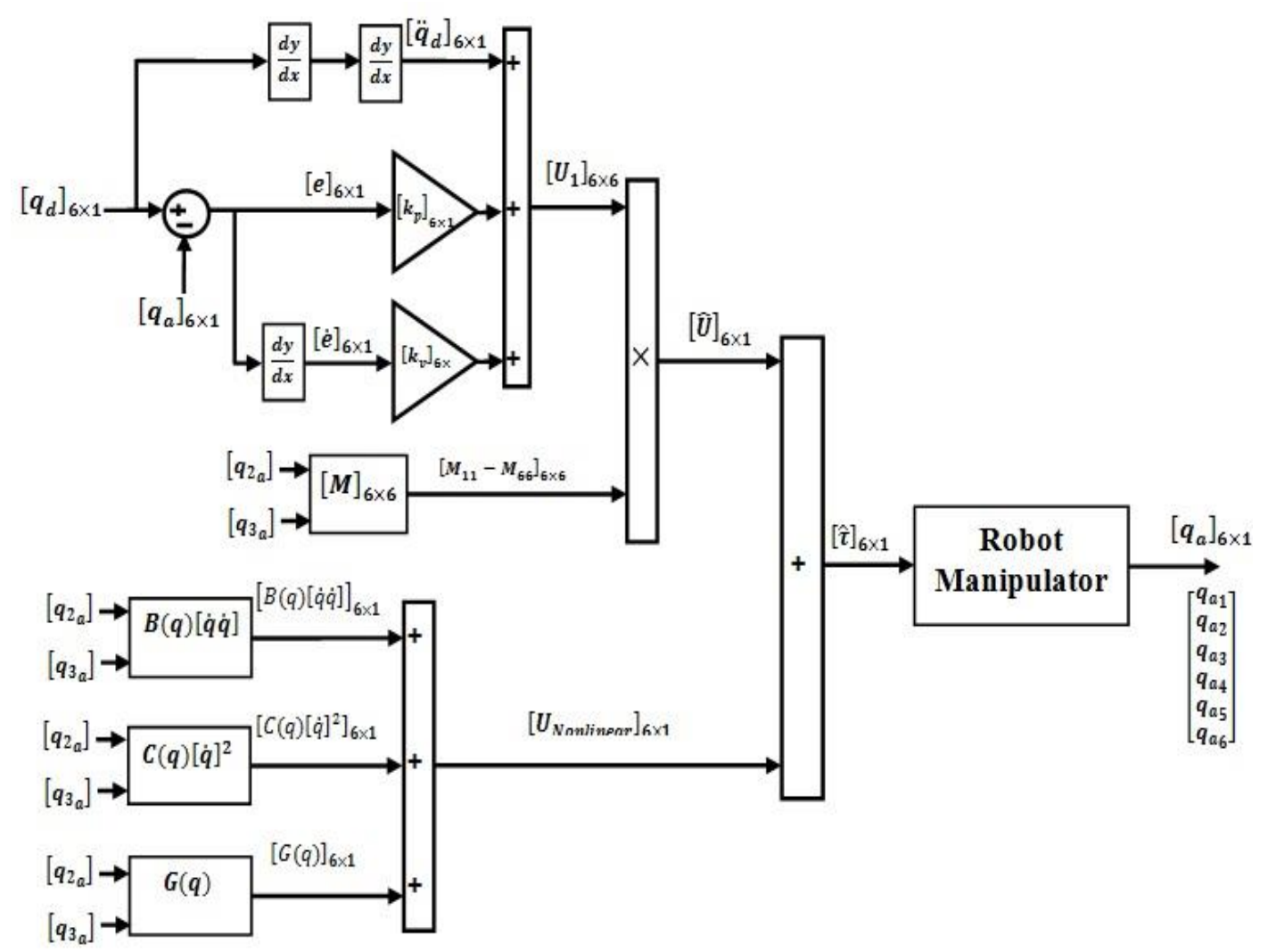

Figure 8. Block Diagram of PD-Computed Torque Controller (PD-CTC)

\section{Test and Result}

In this part two types of controllers are compared, namely; conventional computed torque controller and PD controller. These controllers are tested in certain and uncertain situation.

Comparison of the Tracking Data and Information: the trajectory following of 4 DOF for conventional computed torque controller and PD controller are compared in this section. According to Figure 9, the performance of computed torque controller and PD controller are good enough in certain condition. In rise time point of view, in some joints PD controller is faster than computed torque controller. In error point of view, computed torque controller is better than PD controller. 


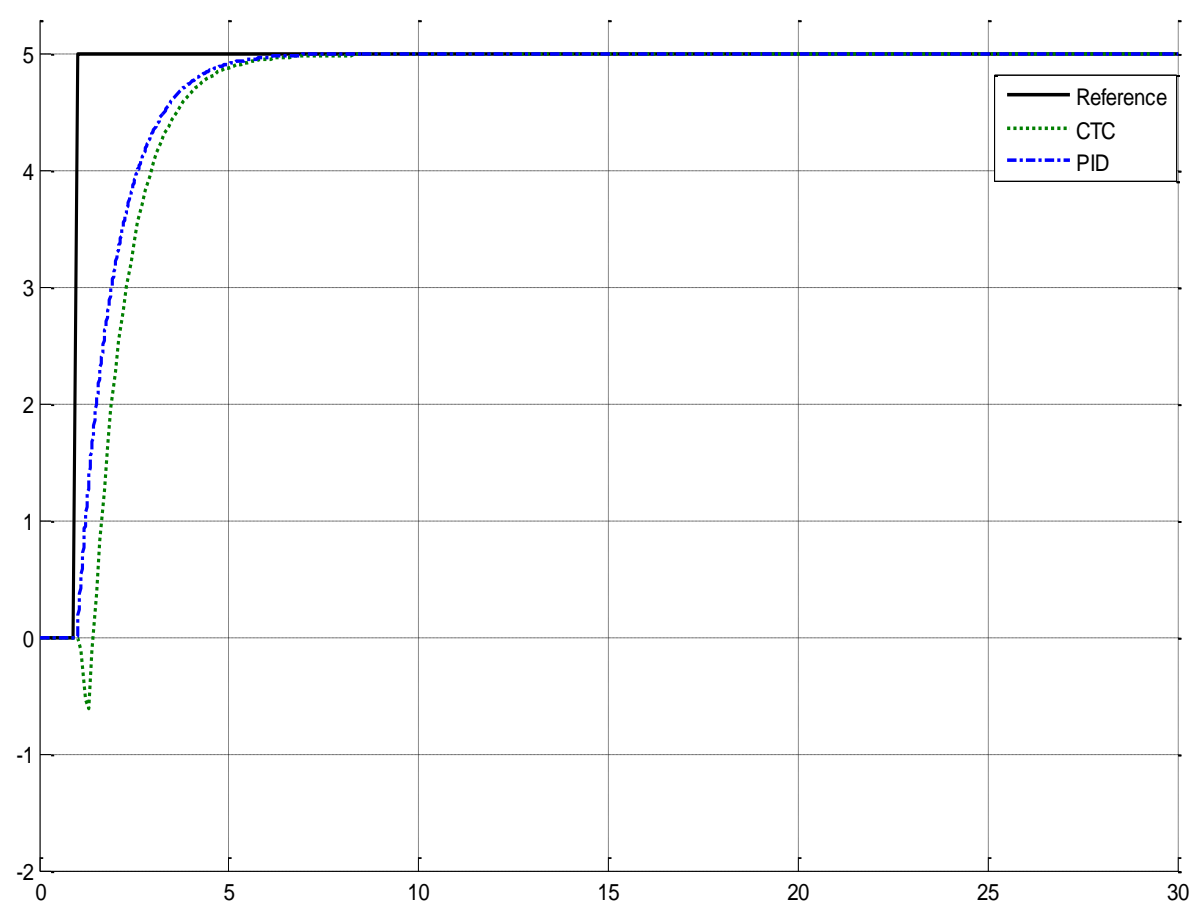

Figure 9. Tracking Data: Conventional CTC and PD Controller

Comparison the actuation $\operatorname{torque}\left(\boldsymbol{\tau}_{\boldsymbol{i}}\right)$ : the control input, forces the robotic manipulators to track the desired trajectories. Figure 10 shows the torque performance in conventional computed torque controller and PD controller. According to the following graph, computed torque controller has steady stable torque performance.

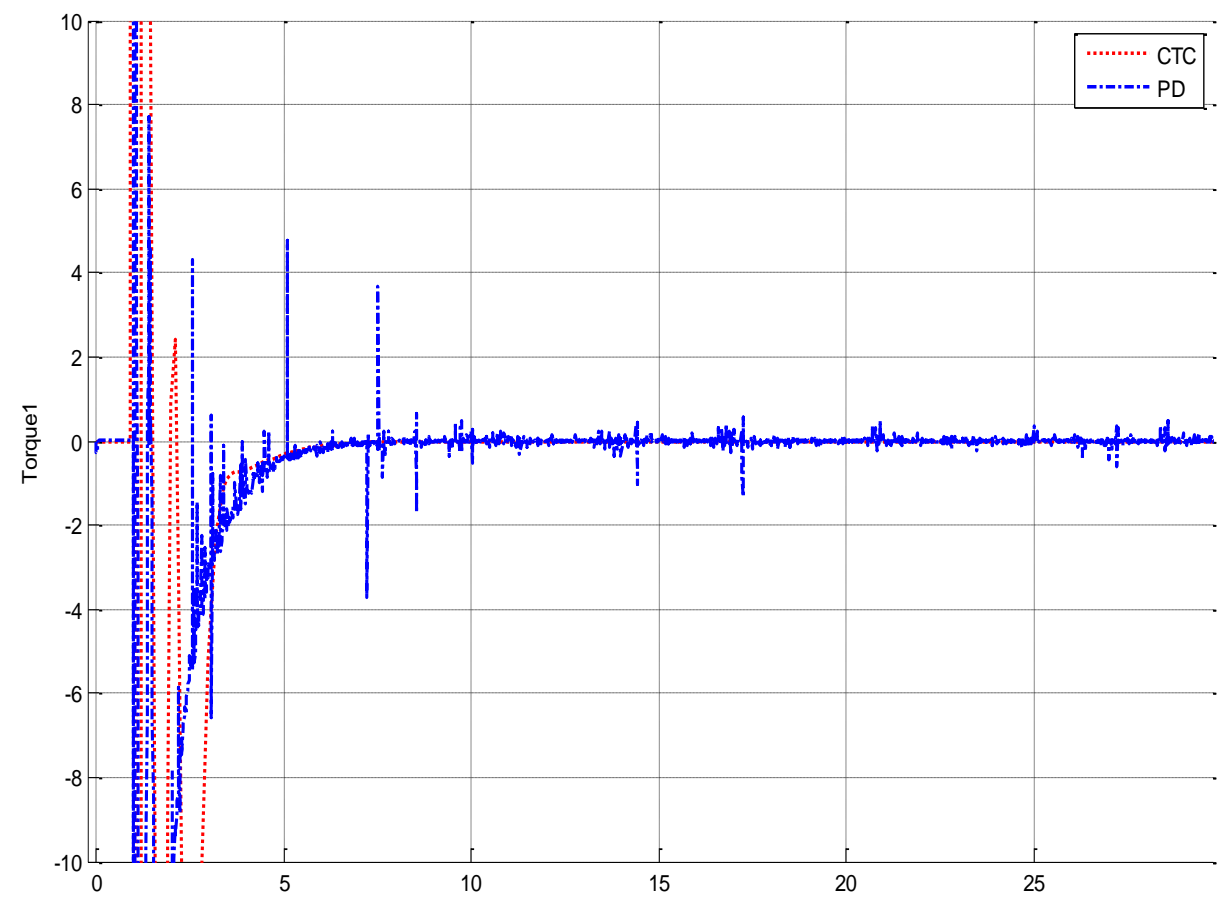

Figure 10. Torque Performance: Conventional CTC and PD Controller 
In the control forces, smaller amplitude means less energy. According to Figure 10, the amplitude of the control forces in PD controller is much larger than CTC. Therefore, CTC require less energy than the PD controller.

Comparison the disturbance rejection: the power of disturbance rejection is very important to robust checking in any controllers. In this section trajectory accuracy, and torque performances are test under uncertainty condition. To test the disturbance rejection band limited white noise with $30 \%$ amplitude is applied to conventional computed torque controller and PD controller. In Figures 11 and 12, trajectory accuracy and torque performance are shown.

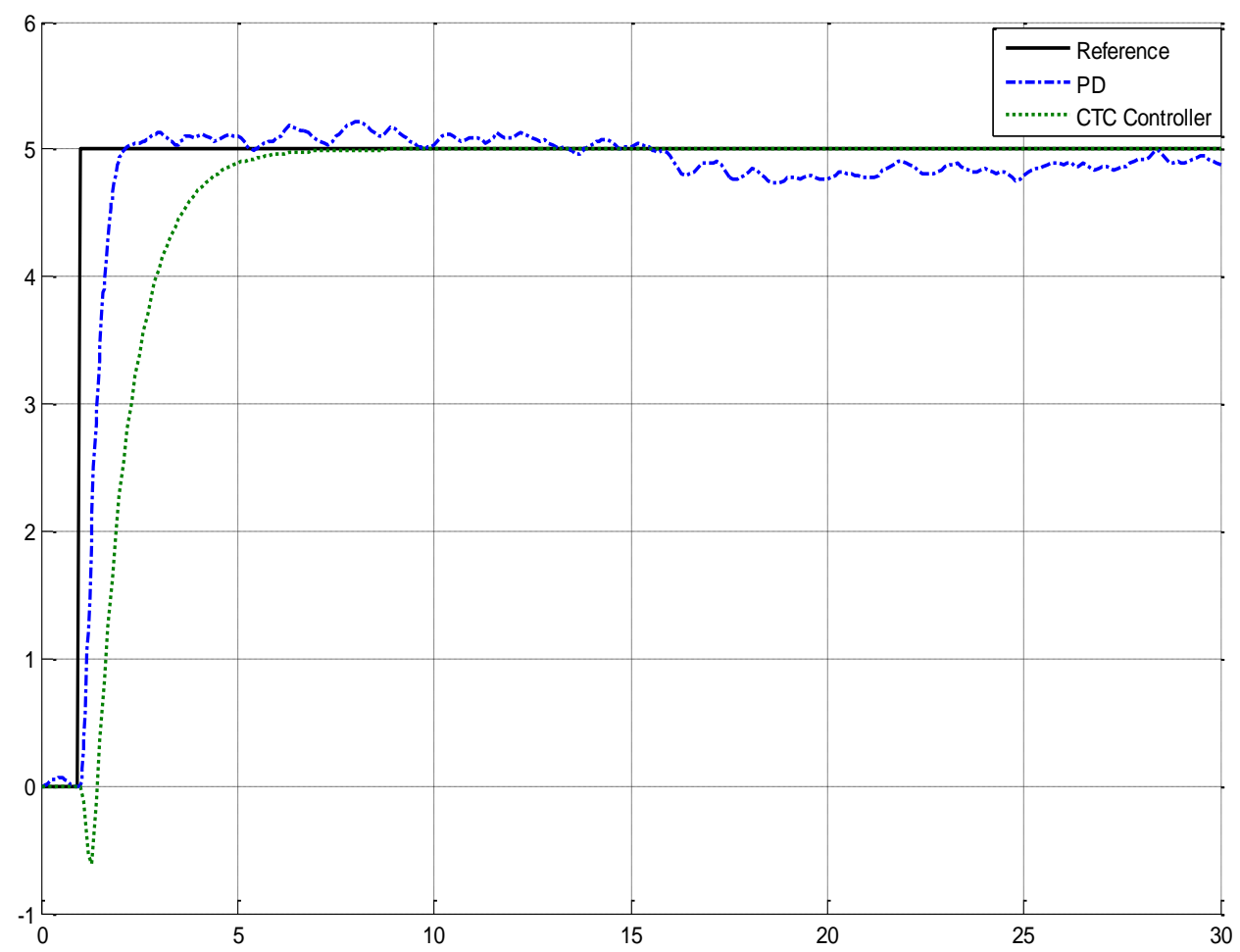

Figure 11. Tracking Data: Conventional CTC and PD Controller in Presence Of Uncertainty

According to above graph, however conventional computed torque controller has rise time in presence of uncertainty but it is more robust than PD controller. PD controller has very much fluctuation in presence of external disturbance. Figure 12 shows the torque performance in presence of uncertainty. 


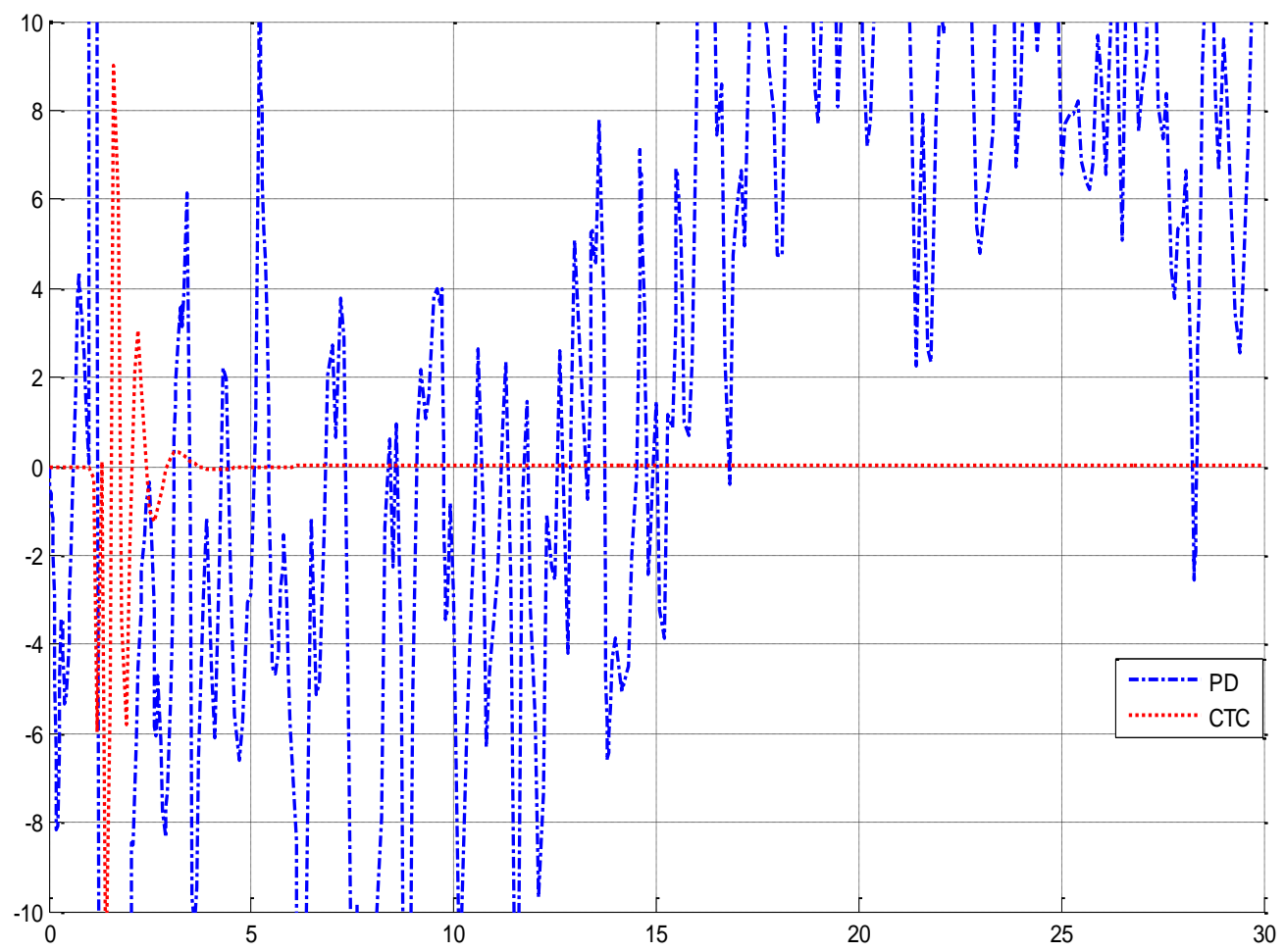

Figure 12. Torque Performance: Conventional CTC and PD Controller in Presence of Uncertainty

After applied uncertainties the force amplitude in PD are increased which will lead to high energy consumption.

\section{Conclusion}

Computed torque controller is a model base nonlinear controller. This controller has many advantages compare to linear controller. In this research, computed torque controller is used to control of surgical robot manipulator. The principles of this type of controller are based on feedback linearization and compute the required arm torques. Computed torque controller works based on behavior (dynamic formulation) of robot manipulator, which caused to works very well when all dynamic and physical parameters are known. In uncertain dynamic parameters when the robot manipulator has variation computed torque controller has challenges but this type of controller's performance is more better than linear controller.

\section{Acknowledgment}

This work was supported by the Iranian Institute of Advance Science and Technology Program of Iran under grant no. 2015-Persian Gulf-2.

Project Title: Research on Intelligent FPGA-Based Algorithm for Four Degrees of Freedom Joints

Iranian center of Advance Science and Technology (IRAN SSP) is one of the independent research centers specializing in research and training across of Control and Automation, Electrical and Electronic Engineering, and Mechatronics \& Robotics in Iran. At IRAN SSP research center, we are united and energized by one mission to discover and develop innovative engineering methodology that solve the most important challenges in field of advance science and technology. The IRAN SSP Center is instead to 
fill a long standing void in applied engineering by linking the training a development function one side and policy research on the other. This center divided into two main units:

- $\quad$ Education unit

- Research and Development unit

Please follow IRANSSP research and training group in: http://iranssp.org/english/

\section{References}

[1] F. Piltan, N. Sulaiman, A. Jalali and F. Danesh Narouei, "Design of Model Free Adaptive Fuzzy Computed Torque Controller: Applied to Nonlinear Second Order System", International Journal of Robotics and Automation, vol. 2, no. 4, (2011), pp. 245-257.

[2] F. Piltan, M. H. Yarmahmoudi, M. Shamsodini, E. Mazlomian and A. Hosainpour, "PUMA-560 Robot Manipulator Position Computed Torque Control Methods Using MATLAB/SIMULINK and Their Integration into Graduate Nonlinear Control and MATLAB Courses", International Journal of Robotics and Automation, vol. 3, no. 3, (2012), pp.167-191.

[3] M. Mirshekaran, F. Piltan, N. Sulaiman, A. Salehi, M. Kazeminasab and Z. Esmaeili, "Hand Tremors Reduction Based on Integral Intelligent Filter Computed Torque Controller", International Journal of Bio-Science and Bio-Technology, vol. 7, no. 1, (2015), pp. 105-120, http://dx.doi.org/10.14257/ijbsbt.2015.7.1.11. (Scopus, SJR=0.24, Q3).

[4] A. Vivas and V. Mosquera, "Predictive functional control of a PUMA robot", International Conference on Automatic Control, pp. 35-40, (2005).

[5] F. Piltan, A. Taghizadegan and N. Sulaiman, "Modelling and Control of Four Degrees of Freedom Surgical Robot manipulator Using MATLAB/SIMULINK", International Journal of Hybrid Information Technology, vol. 8, no. 11, (2015), pp. 47-78, http://dx.doi.org/10.14257/ijhit.2015.8.11.05.

[6] B. Siciliano and O. Khatib, (Eds.), Springer handbook of robotics. Springer Science \& Business Media, (2008).

[7] A. Taghizadegan, F. Piltan, and N. Sulaiman, "Design High Frequency Surgical Robot Controller: Design FPGA-Based Controller for Surgical Robot Manipulator Simscape Modeling", International Journal of Hybrid Information Technology, vol. 9, no. 5, (2016), pp. 431-474. http://dx.doi.org/10.14257/ijhit.2016.9.5.37.

\section{Authors}
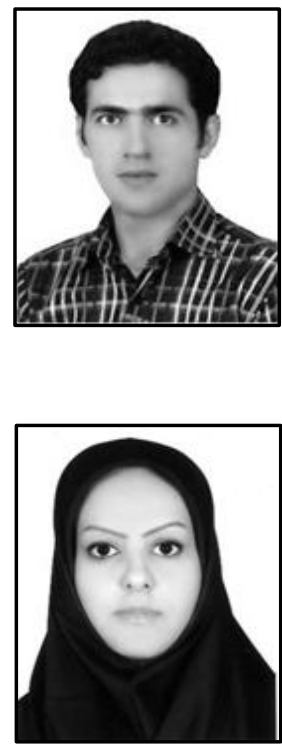

Rouhollah Bahram, he is currently research student at Institute of Advanced Science and Technology, Research Center, IRAN SSP. He is research student of team (6 researchers) to design Micro-electronic Based nonlinear controller for Four Degrees of Freedom Surgical Robot Manipulator since August 2015. His current research interests are nonlinear control, artificial control system, Microelectronic Device, and HDL design.

Samira Soltani, she is currently senior research assistant at Institute of Advance Science and Technology, Research and Development Center, IRAN SSP. She is research assistant of team (47 researchers) to design and build of nonlinear control of industrial robot manipulator for experimental research and education and published about 5 Papers in this field since 2010 to 2012, research assistant (9 researchers) to design and implement intelligent tuning the rate of fuel ratio in internal combustion engine for experimental research and education and published 1 Journal papers since 2011 to 2013, research assistant (34 researchers) of filtering the hand tremors in flexible surgical robot for experimental research and education and published 2 journal papers in this field since 2012 to date, research assistant (21 

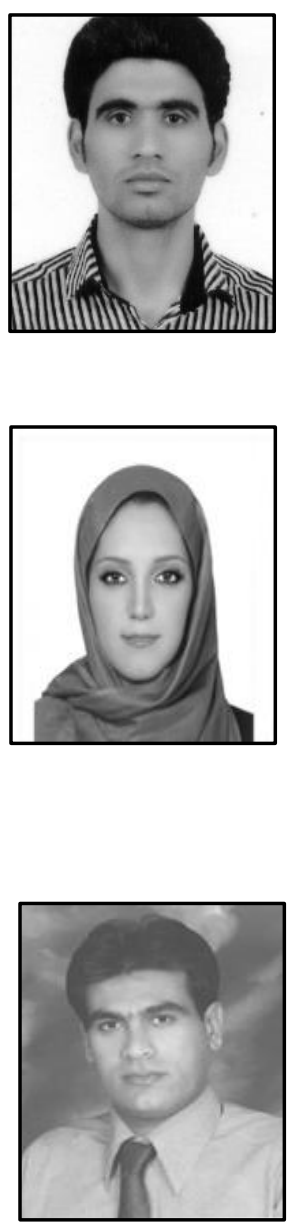

researchers) to design high precision and fast dynamic controller for multi-degrees of freedom actuator for experimental research and education and published 1 journal papers in this field since 2013 to date, research assistant (22 researchers) to research of full digital control for nonlinear systems (e.g., Industrial Robot Manipulator, IC Engine, Continuum Robot, and Spherical Motor) for experimental research and education and published 2 journal papers in this field since 2010 to date, and published more than 12 journal papers since 2011 to date. Her current research interests are nonlinear control, artificial control system and applied to FPGA, and IC engine modeling and control.

Hossein Rashidi Bod, he is currently a research student at Institute of Advanced Science and Technology, Research Center, IRAN SSP. He is research student of team (6 researchers) to design Micro-electronic Based nonlinear controller for Four Degrees of Freedom Surgical Robot Manipulator since August 2015. His current research interests are nonlinear control, artificial control system, Microelectronic Device, and HDL design.

Somayeh Jowkar, she is currently a research assistant at Institute of Advanced Science and Technology, Research Center, IRAN SSP. She is research assistant of team to Design Intelligent FPGA-Based Control Unit to Control of 4-DOF Medical Robot Manipulator since July, 2015 to now, research student (21 researchers) to design high precision and fast dynamic controller for multi-degrees of freedom actuator since 2014 to date, and published 2 journal papers since 2014 to date. Her current research interests are nonlinear control, artificial control system, Microelectronic Device and HDL design.

Amirzubir Sahamijoo, he is currently a senior research assistant at Institute of Advanced Science and Technology, Research Center, IRAN SSP. He is senior research assistant of team to Design Intelligent FPGA-Based Control Unit to Control of 4-DOF Medical Robot Manipulator since July, 2015 to now, research assistant of team ( 8 researchers) to design a Microelectronic Based nonlinear controller for first order delay system since March, 2015 to now, research student (21 researchers) to design high precision and fast dynamic controller for multi-degrees of freedom actuator since 2014 to date, research student (9 researchers) to design Prevent the Risk of Lung Cancer Progression Based on Fuel Ratio Optimization since 2014 to date, and published 4 journal papers since 2014 to date. His current research interests are nonlinear control, artificial control system, Microelectronic Device, Internal Combustion Engine, and HDL design. 

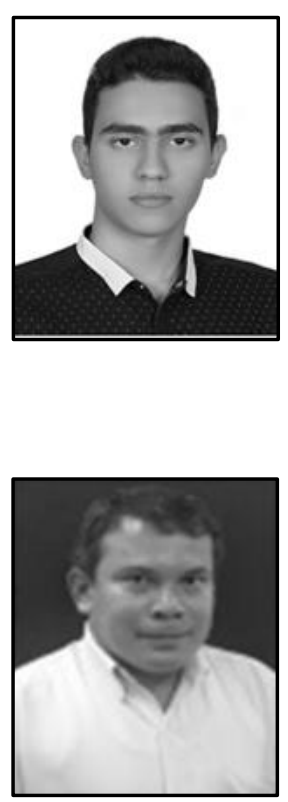

Ali Taghizadegan, he is currently studying as a student in the second grade of Shahid dastgheib's 1 high school and Research Student at Iranian Institute of Advanced Science and Technology, Research and Training Center, IRAN SSP. He is research student of team (6 researchers) to design Micro-electronic Based nonlinear controller for Four Degrees of Freedom Surgical Robot Manipulator since August 2015. His current research interests are nonlinear control, artificial control system, Microelectronic Device, and HDL design.

Nasri Sulaiman, he is a advisor and supervisor of several high impact projects involving more than 150 researchers from countries around the world including Iran, Malaysia, Finland, Italy, Germany, South Korea, Australia, and the United States. Dr. Nasri Sulaiman has authored or co-authored more than 80 papers in academic journals, conference papers and book chapters. His papers have been cited at least 3000 times by independent and dependent researchers from around the world including Iran, Algeria, Pakistan, India, China, Malaysia, Egypt, Columbia, Canada, United Kingdom, Turkey, Taiwan, Japan, South Korea, Italy, France, Thailand, Brazil and more. Dr. Nasri Sulaiman has employed his remarkable expertise in these areas to make outstanding contributions as detailed below:

- Design of a reconfigurable Fast Fourier Transform (FFT) Processor using multi-objective Genetic Algorithms (2008-UPM)

- Power consumption investigation in reconfigurable Fast Fourier Transform (FFT) processor (2010-UPM)

- Crest factor reduction And digital predistortion Implementation in Orthogonal frequency Division multiplexing (ofdm) systems (2011-UPM)

- High Performance Hardware Implementation of a MultiObjective Genetic Algorithm, (RUGS), Grant amount RM42,000.00, September (2012-UPM)

- Nonlinear control for industrial robot manipulator (2010-IRAN SSP)

- Intelligent Tuning The Rate Of Fuel Ratio In Internal Combustion Engine (2011-IRANSSP)

- Design High Precision and Fast Dynamic Controller For MultiDegrees Of Freedom Actuator (2013-IRANSSP)

- Research on Full Digital Control for Nonlinear Systems (2011IRANSSP)

- Micro-Electronic Based Intelligent Nonlinear Controller (2015IRANSSP)

- Active Robot Controller for Dental Automation (2015IRANSSP)

- Design a Micro-Electronic Based Nonlinear Controller for First Order Delay System (2015-IRANSSP) 